\title{
Prostate volume estimations using magnetic resonance imaging and transrectal ultrasound compared to radical prostatectomy specimens
}

\author{
Nicholas R. Paterson, MD;1 Luke T. Lavallée, MD, ;,2 Laura N. Nguyen, MD;1 Kelsey Witiuk, MSc,2 \\ James Ross, MD; Ranjeeta Mallick, PhD,; Wael Shabana, MD, Blair MacDonald, MD, ${ }^{3}$ Nicola Scheida, MD, ${ }^{3}$ \\ Dean Fergusson, PhD,2 Franco Momoli, MD,; Sonya Cnossen, MSc,; Christopher Morash, MD;1 \\ Ilias Cagiannos, MD,1 Rodney H. Breau, MD ${ }^{1,2}$
}

'Division of Urology, Department of Surgery, The Ottawa Hospital, University of Ottawa, Ottawa, ON, Canada; ${ }^{2}$ Ottawa Hospital Research Institute, Department of Clinical Epidemiology, Ottawa, ON, Canada; ${ }^{3}$ Department of Radiology, The Ottawa Hospital, University of Ottawa, Ottawa, ON, Canada

Cite as: Can Urol Assoc J 2016;10(7-8):264-8. http://dx.doi.org/10.5489/cuaj.3236

\section{Abstract}

Introduction: We sought to evaluate the accuracy of prostate volume estimates in patients who received both a preoperative transrectal ultrasound (TRUS) and magnetic resonance imaging $(\mathrm{MRI})$ in relation to the referent pathological specimen post-radical prostatectomy.

Methods: Patients receiving both TRUS and MRI prior to radical prostatectomy at one academic institution were retrospectively analyzed. TRUS and MRI volumes were estimated using the prolate ellipsoid formula. TRUS volumes were collected from sonography reports. MRI volumes were estimated by two blinded raters and the mean of the two was used for analyses. Pathological volume was calculated using a standard fluid displacement method.

Results: Three hundred and eighteen (318) patients were included in the analysis. MRI was slightly more accurate than TRUS based on interclass correlation ( 0.83 vs. 0.74$)$ and absolute risk bias (higher proportion of estimates within 5, 10, and 20 cc of pathological volume). For TRUS, 87 of 298 (29.2\%) prostates without median lobes differed by $>10$ cc of specimen volume and 22 of $298(7.4 \%)$ differed by $>20$ cc. For MRI, 68 of $298(22.8 \%)$ prostates without median lobes differed by $>10 \mathrm{cc}$ of specimen volume, while only 4 of 298 (1.3\%) differed by $>20$ cc.

Conclusions: MRI and TRUS prostate volume estimates are consistent with pathological volumes along the prostate size spectrum. MRI demonstrated better correlation with prostatectomy specimen volume in most patients and may be better suited in cases where TRUS and MRI estimates are disparate. Validation of these findings with prospective, standardized ultrasound techniques would be helpful.

\section{Introduction}

Prostate volume estimates are routinely reported with pelvic imaging. Volume estimates may be useful for radical prostatectomy, brachytherapy, and benign prostate surgery planning. ${ }^{1-6}$ Prostate volume can also be used for calculating prostate-specific antigen (PSA) density when selecting active surveillance candidates. ${ }^{2}$ Volume estimation using other means, such as digital rectal exam or PSA are inaccurate. ${ }^{7-8}$

Studies evaluating the accuracy of transrectal ultrasound (TRUS) for estimating prostate volume have reported mixed results. ${ }^{1,3,5,8,9}$ Some studies have reported that TRUS underestimates prostate volume, while others have found that TRUS overestimates prostate volume. ${ }^{1,4,5,8,9} \mathrm{~A}$ small number of studies have evaluated the accuracy of magnetic resonance imaging (MRI) prostate volume estimates. MRI is proposed to be more accurate than TRUS, but has overestimated prostate volume in most studies. ${ }^{5,6,9}$ Two smaller-powered studies were also found to have mixed results when comparing volume estimations from TRUS and MRI using pathological specimens as the referent standard. 5,9

An increasing number of patients are receiving both TRUS and MRI and may have prostate volume estimates that are discordant between these two methods. In these situations, the imaging modality that provides the most accurate volume is unknown. The purpose of this study was to determine the accuracy of preoperative TRUS and MRI prostate volume estimates by comparing them to radical prostatectomy pathological volume.

\section{Methods}

\section{Patient selection}

The Ottawa Hospital institutional review board approved this study (protocol \#20130779-01H). Patients who received open, laparoscopic, or robotic-assisted radical prostatectomy at The Ottawa Hospital between January 1, 2003 and October 31, 2013 were eligible. To be included, patients must have received both preoperative TRUS and MRI. Clinical information was obtained retrospectively from a prospectively populated database. 


\section{TRUS prostate volume measurements}

TRUS volume measurements were obtained from the most recent sonography report prior to surgery. The prolate ellipsoid formula (height $\mathrm{x}$ width $\mathrm{x}$ length $\mathrm{x} \pi / 6$ ) was used to calculate volume. ${ }^{3}$ TRUS reports were completed prior to MRI imaging, therefore readers were not biased by MRI findings. Complete ultrasound images were not always available for review in our picture archiving and communications system (PACS), therefore repeat measurements by multiple raters was not possible.

\section{MRI prostate volume measurements}

MRI was performed using a 3-Tesla or 1.5-Tesla wholebody MRI (Siemens Symphony, Malvern, PA, U.S.), MR 2004A software, and a pelvic phased-array surface coil. T2-weighted images were obtained in the axial, sagittal, and coronal planes. Two trained investigators blinded to the ultrasound and prostatectomy volumes retrospectively measured MRI prostate dimensions independently and calculated prostate volumes using the prolate ellipsoid formula (height $\mathrm{x}$ width $\mathrm{x}$ length $\mathrm{x} \pi / 6){ }^{3}$. Prostate width was measured in the T2 axial view and the length and height were measured in the T2 mid-sagittal view, as recommended by previous evidence. ${ }^{9}$ The mean of the two raters' MRI volume estimates was used for analyses. The presence of a prominent median lobe $>5 \mathrm{~mm}$ on MRI was determined using the T2 mid-sagittal view and was considered positive if both raters independently noted this finding.

\section{Pathological prostate volume measurements}

Pathological volumes were collected from radical prostatectomy pathology reports. Volumes were determined using standardized pathological techniques by measuring the volume of water displaced by the prostate after removal of peri-prostatic tissue. Prostate weight and volume are highly correlated, ${ }^{10}$ therefore prostate weight was also abstracted in all patients. If prostate volume was not reported, prostate weight was used instead.

\section{Data analysis}

Inter-rater agreement in MRI linear prostate dimensions and volume were assessed using interclass correlation coefficients (ICC). ICC can range from $-1-1$, with 0 representing no correlation, +1 being a perfect positive correlation, and -1 being a perfect negative correlation. We determined if imaging volume estimates were consistently biased by subtracting each pathological volume from imaging estimates to calculate the absolute volume bias. Results were stratified by presence or absence of a median lobe. The accuracy of volume estimates was characterized by calculating proportion of patients whose TRUS and MRI volume estimates differed by greater than 5,10 , or 20 cc of pathological volume.

Covariate analyses were performed to determine if linear associations existed between patient, tumour, and imaging characteristics with pathological prostate volume. Covariates evaluated included patient height and weight, body mass index (BMI), pathological tumour stage, Gleason score, preoperative PSA, and year the imaging was performed.

\section{Results}

During the study period, 2117 patients received radical prostatectomy at The Ottawa Hospital (Fig. 1). Of those, $318(15.0 \%)$ patients had both preoperative TRUS and MRI performed. Mean patient age at surgery was 62.7 years (standard deviation [SD] 7.3), BMI was 28.2 (SD 4), and PSA was 9.4 (SD 8.3). Mean prostate volume from TRUS, MRI, and pathological measurements were 34.4 cc (SD 18.3), 39.2 cc (SD 17.8), and 37.3 cc (SD 15.9), respectively. A prominent median lobe was present in $20(6 \%)$ patients. In $34(11 \%)$ pathology reports, prostate volume was not reported and prostate weight was used instead (interclass correlation coefficient between prostate volume and prostate weight was $0.96 ; p<0.0001$ for the 284 patients that had both measurements documented).

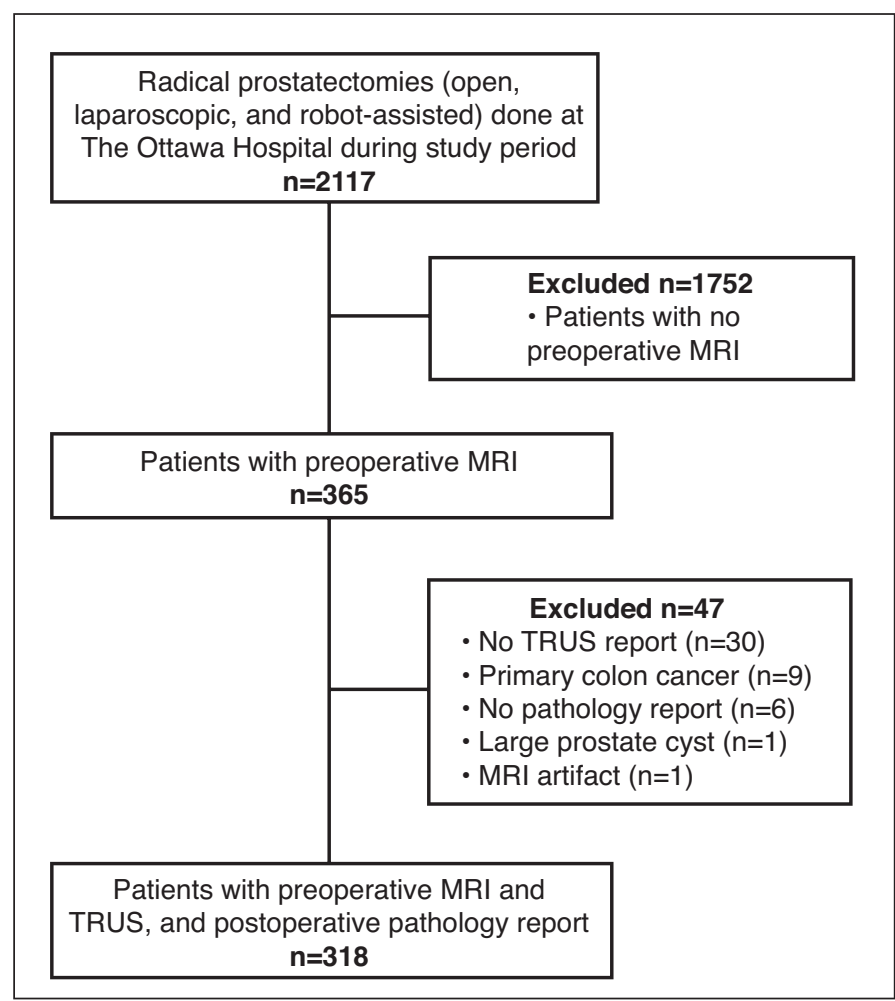

Fig. 1. Flow chart of patients included/excluded from study cohort. MRI: magnetic resonance imaging; TRUS: transrectal ultrasound. 


\begin{tabular}{|c|c|c|c|c|}
\hline \multirow[b]{2}{*}{$\begin{array}{l}\text { Volume } \\
\text { difference } \\
\text { (cc) }\end{array}$} & \multicolumn{2}{|c|}{ TRUS } & \multicolumn{2}{|c|}{ MRI } \\
\hline & $\begin{array}{c}\text { No median } \\
\text { lobe }(n=298), \\
n(\%)\end{array}$ & $\begin{array}{c}\text { Median } \\
\text { lobe }(n=20) \\
n(\%)\end{array}$ & $\begin{array}{c}\text { No median } \\
\text { lobe }(n=298), \\
n(\%)\end{array}$ & $\begin{array}{c}\text { Median } \\
\text { lobe }(n=20), \\
n(\%)\end{array}$ \\
\hline$>5$ & $176(59.1)$ & $15(75.0)$ & $168(56.4)$ & $12(60.0)$ \\
\hline$>10$ & $87(29.2)$ & $10(50.0)$ & $68(22.8)$ & $8(40.0)$ \\
\hline$>20$ & $22(7.4)$ & $5(25.0)$ & $4(1.3)$ & $4(20.0)$ \\
\hline
\end{tabular}

\section{Inter-rater reliability of investigators MRI prostate volume measurements}

There was agreement between the two the independent raters of MRI prostate volume. Volume estimates calculated using the prolate ellipsoid formula had an ICC of 0.91 .

\section{Accuracy of TRUS prostate volume measurements}

The ICC for TRUS and pathological volume was $0.74(95 \%$ confidence interval [CI] 0.71-0.80). The mean TRUS volume underestimated prostate volume by 3.4 cc (Fig. 2A). For TRUS, 87 of 298 (29.2\%) prostates without median lobes differed by $>10 \mathrm{cc}$ of specimen volume and 22 of $298(7.4 \%)$ differed by $>20$ cc (Table 1$)$. In prostates with median lobes, $25 \%(n=5)$ had $>20$ cc discrepancy from the specimen volume.

\section{Accuracy of MRI prostate volume measurements}

The ICC for MRI and pathological volume was $0.83(95 \% \mathrm{Cl}$ 0.79-0.93). MRI overestimated prostate volume by a mean

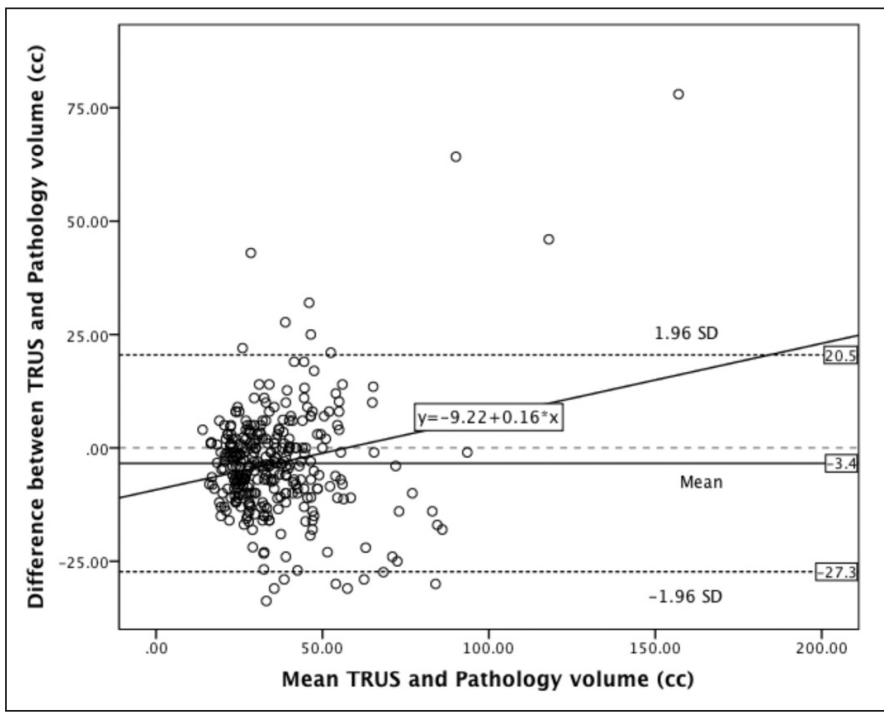

Fig. 2A. Bland-Altman plot showing the agreement between transrectal ultrasound (TRUS) and pathological volume estimates. of $1.4 \mathrm{cc}$ (Fig. 2B). This overestimation was magnified in the cohort identified to have a prominent median lobe $(n=20)$ (mean overestimation 6.9 cc). For MRI, 68 of 298 (22.8\%) prostates without median lobes differed by $>10 \mathrm{cc}$ of specimen volume, while only four of $298(1.3 \%)$ differed by $>20$ cc (Table 1). In prostates with median lobes, $20 \%(n=4)$ had $>20$ cc discrepancy from the specimen volume.

\section{Agreement between MRI and TRUS}

Prostate volume estimations from TRUS and MRI showed high agreement (Fig. 2C). Eighty-seven (87) (27\%) patients had a 10-20 cc difference between MRI and TRUS estimates, while $15(5 \%)$ had a difference $>20$ cc. When the difference was between 10 and 20cc, MRI estimated pathological specimen volume more accurately than TRUS in $63 \%$ of cases. When the difference was $>20 \mathrm{cc}$, MRI was more accurate in $73 \%$ of cases. Presence of a median lobe decreased the ability for TRUS and MRI to accurately estimate prostate volume (Table 2).

\section{Patient and tumour characteristics and prostate volume}

Multivariable analyses of baseline patient characteristics showed no statistically significant associations between patient, tumour, and imaging characteristics with pathological volume measurements, with the exception of an intravesical median lobe (Table 2).

\section{Discussion}

This is the largest study to date assessing prostate volume using MRI and TRUS compared to prostate volumes from pathological specimens. Overall, MRI and TRUS provide accurate estimates of prostate volume for most patients. ${ }^{2}$ If TRUS and MRI estimates are disparate in an individual patient, it seems that MRI findings are more likely to be correct. MRI was slightly more accurate than TRUS based on interclass correlation (0.83 vs. 0.74$)$ and absolute risk bias (higher proportion of estimates within 5, 10, and 20 cc of pathological volume). On average, MRI slightly overestimated prostate volume (mean $1.4 \mathrm{cc}$ ) and TRUS slightly underestimated prostate volume (mean -3.4 cc). The presence of a prominent median lobe decreased the accuracy of both MRI and TRUS.

Our finding that MRI overestimates prostate specimen volume is consistent with previous studies. ${ }^{5,9}$ One study comparing 21 prostatectomy specimens to TRUS and MRI found that MRI had better correlation with pathology, but that the accuracy of volume estimates depended on the views (axial vs. sagittal) used for measurements. ${ }^{9}$ Another study comparing 73 prostatectomy specimens with preoperative imaging found that MRI overestimated smaller prostates $(<35 \mathrm{cc})$ and underestimated larger prostates $(>35 \mathrm{cc}) .{ }^{5}$ Reports on 


\begin{tabular}{|c|c|c|c|c|c|c|}
\hline \multirow[t]{2}{*}{ Covariate } & \multicolumn{3}{|c|}{ MRI } & \multicolumn{3}{|c|}{ TRUS } \\
\hline & Co-efficient & (95\% Cl) & p value & Co-efficient & (95\% Cl) & p value \\
\hline $\mathrm{BMI}$ & 0.105 & $(-0.004-0.215)$ & 0.060 & 0.072 & $(-0.063-0.207)$ & 0.296 \\
\hline Height & 0.098 & $(-0.091-0.287)$ & 0.310 & -0.078 & $(-0.317-0.162)$ & 0.523 \\
\hline Weight & 0.010 & $(-0.077-0.096)$ & 0.830 & 0.019 & $(-0.089-0.128)$ & 0.729 \\
\hline Median lobe & -8.123 & $(-12.766-3.840)$ & 0.001 & -8.150 & $(-13.880--2.421)$ & 0.005 \\
\hline pT3 (vs. pT2) & -1.789 & $(-4.082-0.504)$ & 0.126 & -2.169 & $(-4.989-0.650)$ & 0.131 \\
\hline Gleason sum (6 vs. 7 vs. $8-10$ ) & 1.157 & $(-4.082-0.504)$ & 0.084 & 0.725 & $(-0.895-2.344)$ & 0.380 \\
\hline Preoperative PSA & 0.088 & $(-0.043-0.218)$ & 0.187 & 0.115 & $(-0.049-0.279)$ & 0.168 \\
\hline Year of MRI/TRUS & 0.465 & $(-0.052-0.981)$ & 0.078 & 0.062 & $(-0.574-0.699)$ & 0.847 \\
\hline
\end{tabular}

whether TRUS over or underestimates prostate specimen volume are more inconsistent. ${ }^{1,4,5,8,9}$ Our findings suggest TRUS underestimates prostate volume relative to both pathological volume and MRI estimates. However, it is important to note that most errors in prostate volume estimation are unlikely to be clinically significant.

The mean volume difference between TRUS and MRI estimations was $4.8 \mathrm{cc}$. While statistically significant, it is not clinically significant for most patients. In a large sample comparing prostate volumes measured by TRUS and MRI that were not compared to pathology, the average volume difference was only $1.7 \mathrm{cc}^{2}$ In our cohort, $6 \%$ of patients had a prominent median lobe, which resulted in volume inaccuracy. Our data suggest that prostates with MRI-confirmed median lobes caused prostate volume overestimation $(+6.9$ in prostates with median lobes vs. $1.1 \mathrm{cc}$ in prostates without median lobes) and increased variability compared to pathological specimens. This phenomenon is likely due to the limitations of the ellipsoid formula in accounting for the non-uniform shape created by the increased height of the median lobe. ${ }^{11}$ Other established methods of estimating

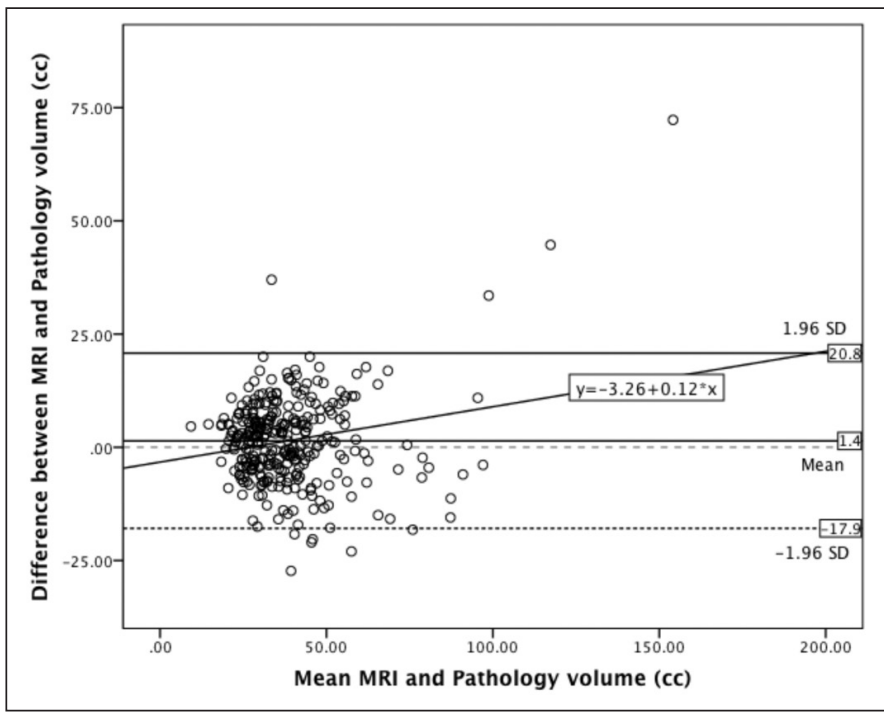

Fig. 2B. Bland-Altman plot showing the agreement between magnetic resonance imaging (MRI) and pathological volume estimates. prostate volume on MRI that take into account non-uniform shapes include: stepwise planimetry, semi-automated and fully automated segmentation. ${ }^{12}$ While methods such as planimetry may be more accurate at estimating prostate volume, the significant time and cost burden associated with manually outlining the prostate on each slice means it is rarely performed in practice..$^{9,11,12}$

There are several potential limitations to our study. Only one measurement was available for both TRUS and pathological volumes, compared to two raters for MRI images, which may have resulted in less accurate TRUS or pathological measurements. The prolate ellipsoid formula may also be less accurate compared to stepwise planimetry or other modalities. ${ }^{9,12}$ Finally, the association between errors in volume estimates and clinical outcomes were not assessed.

\section{Conclusion}

Prostate volume is routinely reported with pelvic imaging. Prostate volumes estimated using TRUS and MRI show

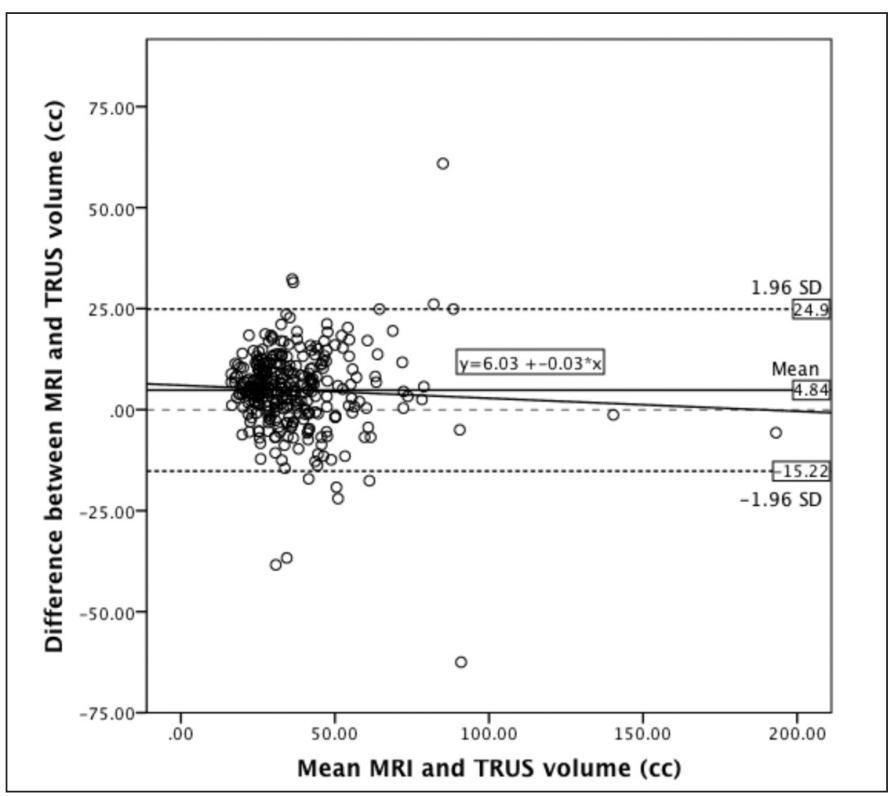

Fig. 2C. Bland-Altman plot showing the agreement between magnetic resonance imaging (MRI) and transrectal ultrasound (TRUS) volume estimates. 
Paterson et al.

excellent agreement with each other and with radical prostatectomy specimens. In our study, MRI demonstrated greater accuracy and may be used in cases where TRUS and MRI estimates differ significantly. The presence of a median lobe may lead to volume overestimation when using the prolate ellipsoid formula.

Competing interests: Dr. Morash has been an Advisory Board member for AbbVie, Astellas, AstraZeneca, Bayer, Janssen, and Sanofi; and has participated in clinical trials for AbbVie. Dr. Cagiannos has been an Advisory Board member for and has received grant support from AbbVie and Ferring. The remaining authors declare no competing financial or personal interests.

This paper has been peer-reviewed.

\section{References}

1. Matthews $G$, Motta J, Fracehia J. The accuracy of transrectal ultrasound prostate volume estimation: Clinical correlations. J Clin Ultrasound 1996; 24:501-5. http://dx.doi.org/10.1002/(SICI) 1097 . 0096(199611/12)24:9<501::AlD-JCU2>3.0.C0;2-R

2. Weiss BE, Wein AJ, Malkowicz SB, et al. Comparison of prostate volume measured by transrectal ultrasound and magnetic resonance imaging: Is transrectal ultrasound suitable to determine which patients should undergo active surveillance? Urol Oncol 2013;31:1436-40. http://dx.doi.org/10.1016/i. urolonc.2012.03.002

3. Tewari A, Induchara R, Shinohara K, et al. Comparison of transrectal ultrasound prostatic volume estimation with magnetic resonance imaging volume estimation and surgical specimen weight in patients with benign prostatic hyperplasia. J Clin Ultrasound 1996;24:169-74. http://dx.doi.org/10.1002/(SICI) 10970096(199605)24:4<169::AID-JCU2>3.0.C0;2-D

4. Nathan MS, Seenivasagam K, Mei $Q$, et al. Transrectal ultrasonography: Why are estimates of prostate volume and dimension so inaccurate? Brit J Urol 1996;77:401-7. hittp://dx.doi.org/10.1046/i.1464410X.1996.90214.x

5. Lee JS, Chung BH. Transrectal ultrasound vs. magnetic resonance imaging in the estimation of prostate volume as compared with radical prosatectomy specimes. Urol Int 2007;78: 323-7. http://dx.doi. org/10.1159/000100836

6. Park H, Kim JY, Lee BM, et al. A comparison of preplan MRI and preplan CT-based prostate volume with intraoperative ultrasound-based prostate volume in real-ime permanent brachytherapy. Radiat Oncol 201;29:199-205. http://dx.doi.org/10.3857/roj.2011.29.3.199

7. Roehrborn CG. Accurate determination of prostate size via digital rectal examination and transrectal ultrasound. Urology 1998;4295:19-22. http://dx.doi.org/10.1016/s0090-4295(98)00051-x

8. Loeb $S$, Han $M$, Roehl KA, et al. Accuracy of prostate weight estimation by digital rectal examination vs. transrectal ultrasonography. J Urol 2005;173:63-5. http://dx.doi.org/10.1097/01. ju.0000145883.01068.5f

9. Jeong CW, Park HK, Hong SK, et al. Comparison of prostate volume measured by transrectal ultrasonography and MRI with the actual prostate volume measured after radical prostatectomy. Urol Int 2008;81:179-85. http://dx.doi.org/10.1159/000144057

10. Varma $M$, Morgan $J M$. The weight of the prostate gland is an excellent surrogate for gland volume. Histopathology 2010;57:55-8. http://dx.doi.org/10.1111/j.1365-2559.2010.03591.x

11. Aarnink R, De La Rosette J, Debruyne F, et al. Reproducibility of prostate volume measurements from transrectal ultrasonography by an automated and manual technique. Brit J Urol 1996;78:219-23. http://dx.doi.org/10.1046/i.1464-410X.1996.08213.x

12. Garvey R, Turkbey $B$, Truong $H$, et al. Clinical value of prostate segmentation and volume determination on MRl in benign prostatic hyperplasia. Diagn Interv Radiol 2014;20:229-33. http://dx.doi.org/10.5152/ dir.2014.13322

Correspondence: Dr. Rodney H. Breau, Ottawa Hospital Research Institute, Division of Urology, Department of Surgery, University of Ottawa, Ottawa, ON, Canada; rbreau@toh.on.ca

\section{Zoladex \\ goserelin $3.6 \mathrm{mg}$}

Indication and clinical use:

Zolade ${ }^{\circledR}$ is indicated for the palliative treatment of patients with hormone-dependent advanced carcinoma of the prostate (Stage M1 or Stage D2) and for use in combination with a non-steroidal antiandrogen and radiation therapy for the management of locally advanced $(\mathrm{T} 3, \mathrm{~T} 4)$ or bulky Stage T2b, T2c carcinoma of the prostate. Zoladex ${ }^{\circledR}$ can be used as adjuvant hormone therapy to external beam irradiation for patients with locally advanced prostate cancer (Stage T3-T4).

Treatment with Zolade ${ }^{\circledR}$ and a non-steroidal antiandrogen should start 8 weeks prior to initiating radiation therapy and continue until completion of the radiation therapy. The safety and effectiveness of Zolade ${ }^{\circledR}$ in children has not been established

\section{Contraindications:}

Hypersensitivity to goserelin/depot or any component of the container

\section{Most serious warnings and precautions:}

Osteoporosis: Assessment of osteoporosis risk and management according to clinical practice and guidelines should be considered.

Tumor flare reaction: Patients at risk of developing ureteric obstruction should be closely monitored during the first month of therapy. Patients with vertebral metastases who are thought to be at particular risk of spinal cord compression should be closely monitored during the first month of treatment.

Injection site injuries and vascular injuries: Patients should be monitored for signs or symptoms of abdominal hemorrhage. Zolade $x^{\circledR}$ is not recommended in patients with low body mass index $(\mathrm{BMl}<18.5)$ or in patients who are fully anticoagulated (INR $>2)$.

Other relevant warnings and precautions:

- Transient elevation of serum testosterone concentrations

- Increased cardiovascular risk factors

- Induced hypogonadism

- Impaired glucose tolerance

- Anemia

- Depression (sometimes severe)

- Pituitary-gonadal suppression

- Use in children has not been established; labeling reflects safety and effectiveness in patients over 65 years of age

- Treatment requires routine monitoring, physical examinations and appropriate laboratory tests

\section{For more information:}

Please consult the Product Monograph for Zoladex ${ }^{\circledR}$ at www.azinfo.ca/zoladex/pm965 for important information relating to adverse reactions, drug interactions and dosing information. The Product Monograph is also available by calling us at 1-800-565-5877. 